\title{
Ensayos clínicos publicados en el Boletín Médico del Hospital Infantil de México: un análisis crítico
}

\author{
Leticia A. Barajas-Nava ${ }^{1 *}$, Andrea Sandoval-Campos ${ }^{2}$ y Karen Aguirre-Raya ${ }^{3}$ \\ ${ }^{1}$ Unidad de Investigación de Medicina Basada en Evidencias, Hospital Infantil de México Federico Gómez, Ciudad de México; ${ }^{2} E s c u e l a ~ d e$ \\ Medicina, Universidad Tominaga Nakamoto, Estado de México; ${ }^{3}$ División de Pediatría, Hospital Juárez de México, Ciudad de México. México
}

\begin{abstract}
Resumen
Introducción: El ensayo clínico es la manera más rigurosa de conducir los experimentos en seres humanos. Desde su introducción en investigación biomédica se han implementado cambios en el modo de establecer las bases para el diagnóstico, pronóstico y la terapéutica en la práctica clínica. Se han realizado estudios para identificar los ensayos clínicos publicados en diferentes áreas médicas, pero hasta el momento ninguno había identificado los ensayos clínicos publicados en el Boletín Médico del Hospital Infantil de México (BMHIM). El objetivo de este trabajo fue identificar y describir los ensayos clínicos controlados (ECC) publicados en el BMHIM. Métodos: Se realizó búsqueda manual y sistemática en cada uno de los números y volúmenes del BMHIM de 1968 a 2016. Se registraron los ECC para obtener sus principales características. Adicionalmente, se evaluó su calidad metodológica mediante la herramienta de riesgo de sesgo. Los resultados se presentan de forma descriptiva, gráfica y temporal. Resultados: Se revisaron 73 números con 363 volúmenes, analizando 4925 artículos. La proporción de ECC identificados en el BMHIM fue del 1\% (67/4925). En general, los ensayos clínicos se realizaron en el contexto nacional, en el tercer nivel de atención, con un tamaño de muestra reducido, y las intervenciones farmacológicas fueron las más utilizadas. La calidad metodológica de los estudios fue baja, con alto riesgo de sesgo. Conclusiones: Los ensayos clínicos representan el 1\% de todos los artículos de investigación originales publicados en el BMHIM. Aún existen áreas de investigación pediátrica, las cuales requieren del desarrollo de ECC para mejorar la práctica clínica, así como para elevar la calidad de la investigación.
\end{abstract}

Palabras clave: Ensayo clínico. Ensayo clínico controlado aleatorizado. Riesgo de sesgo. Calidad.

\section{Clinical trials published in the Boletín Médico del Hospital Infantil de México: a critical analysis}

\begin{abstract}
Background: Controlled clinical trials (CCT) are the study design with the highest accuracy and evidence level. From its introduction in biomedical research, changes have been implemented in the way of establishing the basis for diagnosis, prognosis and treatment in clinical practice. Studies to identify published CCTs regarding different medical fields have been carried out. To date, none of them has identified the clinical trials that have been published in the Boletín Médico del Hospital Infantil de México (BMHIM). The aim of this study was to identify and describe the controlled clinical trials published in
\end{abstract}

Correspondencia:

*Leticia A. Barajas-Nava

E-mail: leticiaa.barajas@gmail.com

DOI: 10.24875/BMHIM.M18000026
Fecha de recepción: 26-07-2017

Fecha de aceptación: 16-12-2017
Disponible en internet: 10-08-2018 Bol Med Hosp Infant Mex. 2018;75:244-254 www.bmhim.com

1665-1146/@ 2018. Hospital Infantil de México Federico Gómez, impreso por Permanyer México SA de CV, todos los derechos reservados. 
the BMHIM. Methods: A manual and systematic search was performed in each of the volumes of the BMHIM from 1968 to 2016. CCTs were recorded to obtain their main characteristics. Additionally, their methodological quality was assessed through the "risk of bias"tool. Results are presented in a descriptive, graphic and time-based manner. Results: In total, 73 issues with 363 volumes were reviewed, and 4925 articles were analyzed. The proportion of CCTs identified in the BMHIM was $1 \%$ (67/4925). In general, clinical trials were performed in the national context and in the third-level of medical care. CCTs also presented reduced sample sizes; pharmacological interventions were the most frequent. The methodological quality of the studies was low with a high risk of bias. Conclusions: Clinical trials represented $1 \%$ of all the original research articles published in the BMHIM. There are still pediatric research fields that require CCTs to be developed in order to improve clinical practice, as well as to increase the quality of the research.

Key words: Clinical trial. Randomized controlled trial. Risk of bias. Quality.

\section{Introducción}

El Hospital Infantil de México Federico Gómez (HIMFG) fue inaugurado el 30 de abril de 1943. Al mismo tiempo, el Dr. Federico Gómez fue nombrado como primer director del hospital, quien afirmó que "el hospital desempeñaría tanto labores asistenciales como funciones de enseñanza e investigación"1-4. El emblema que representaría al hospital fue Ixtlilton, nombre de la lengua náhuatl que en la mitología mexicana representaba a la deidad de la medicina infantil, a quien se le atribuía la facultad de curar y hacer hablar a los niños ${ }^{1,5}$.

La fundación del HIMFG dio inicio a la modernidad del sistema de salud en México, conformando el primero de los actuales Institutos Nacionales de Salud ${ }^{1,6}$.

En el mes de diciembre del mismo año de la inauguración del hospital (1943), inicia la producción del Boletín Médico del Hospital Infantil de México (Bol Med Hosp Infant Mex, BMHIM), pero no fue sino hasta el bimestre de marzo-abril de 1944 que se publica el primer volumen y número del boletín ${ }^{5,7}$.

El BMHIM actualmente cuenta con más de 70 años de experiencia y fue considerado por muchos años el instrumento de divulgación pediátrica de mayor prestigio en el país y el resto de América Latina ${ }^{1,5,8}$. Se realiza como una publicación periódica bimestral, es decir, se publican seis números al año, y es editado por el HIMFG.

El BMHIM recibe trabajos originales en español e inglés, principalmente sobre pediatría y ciencias afines, los cuales pueden ser artículos de investigación, artículos de revisión, casos clínico-patológicos, temas pediátricos de salud pública, cartas al editor y comentarios editoriales por invitación ${ }^{9}$. Desde su inicio y hasta finales del año 2016 se habían emitido 73 volúmenes publicados de manera ininterrumpida.

Actualmente el BMHIM se encuentra indizado en Academic Search Complete de EBSCO, Artemisa, Bibliomex Salud, Free Medical Journals, Imbiomed,
Latindex-catálogo, Latindex-directorio, LILACS (Literatura Latino-Americana e do Caribe en Ciências de Saúde), Medic Latina, Medigraphic, Pascal, Periódica (Índice de Revistas Latinoamericanas de Ciencias), SCIELO, MEDLINE/Pubmed, Emergent Sources Citation Index y Scopus ${ }^{10}$.

El BMHIM es una revista que se encuentra clasificada como Q3 y H6 según el Sistema de Clasificación de Revistas Mexicanas de Ciencia y Tecnología (CONACYT $)^{11}$. El Q3 es considerado como un indicador (cuartil) de medida de posición de una revista en relación con todas las de su área, ordenadas de mayor a menor impacto. El H6 es un indicador que permite evaluar la producción científica de un investigador en función del número de citas, asignándole esta nomenclatura al BMHIM de acuerdo con el promedio de publicaciones de sus autores ${ }^{12}$.

De acuerdo con el sistema de clasificación de Dewey, al BMHIM se le ha asignado el número 610 , correspondiente a Ciencias Médicas (Medicina). El sistema Dewey es una clasificación bibliométrica fundamentalmente enumerativa, de base decimal, ordenada por disciplina y de carácter jerárquico ${ }^{13}$. Asimismo, esta revista se encuentra registrada en Latindex, que es un sistema de información sobre las revistas de investigación científica, técnico-profesionales y de divulgación científica y cultural que se editan en los países de América Latina, el Caribe, España y Portugal, asignándole la nomenclatura ISSN 0539-61159,10.

\section{El ensayo clínico}

El ensayo clínico controlado (ECC) es considerado como la mejor evidencia científica disponible para la toma de decisiones en el ámbito sanitario; es la manera más rigurosa de realizar los experimentos clínicos en seres humanos ${ }^{14-16}$. La importancia que adquirieron estos estudios a lo largo de las últimas décadas ha generado un cambio en la práctica de la medicina ${ }^{17,18}$. 
Sir Richard Doll y Bradford Hill fueron precursores de los primeros ensayos clínicos documentados al demostrar que fumar cigarrillos causaba cáncer de pulmón ${ }^{19,20}$, mientras que el nacimiento de lo que hoy en día conocemos como ensayo clínico aleatorizado tuvo lugar a partir de una publicación del año 1948, generada por el Consejo de Investigación Médica de Reino Unido, donde se analizó y demostró la eficacia de la estreptomicina en el tratamiento de la tuberculosis $^{17-19,21}$. Un desarrollo similar ocurrió paralelamente en Europa y Estados Unidos en la segunda mitad del siglo $\mathrm{XX}$, generando múltiples estudios de valoración terapéutica para numerosas enfermedades como úlcera gástrica, infarto de miocardio y cáncer. Es importante destacar la implementación del cegamiento en los ensayos clínicos, la cual surge en Alemania a partir de intervenciones farmacológicas, lo cual permitió minimizar los sesgos de información en los estudios. Asimismo, el uso del placebo permitió evaluar de manera más objetiva la eficacia y efectividad de los tratamientos ${ }^{19}$.

También es memorable el masivo ensayo clínico de campo realizado en 1954 en Estados Unidos para probar la vacuna de virus vivos atenuados de Jonas Salk contra la poliomielitis, que incluyó 623,972 niños en edad escolar y que puede considerarse uno de los ensayos clínicos más grandes de la historia de la medicina $^{19,22}$.

Es así como surge el ensayo clínico, como un experimento cuidadoso y éticamente diseñado en el que los participantes son asignados a las diferentes modalidades de intervención de manera simultánea, y en algunos casos de forma aleatoria, considerándose así el método epidemiológico para comprobar una hipótesis. Al ser un experimento controlado en voluntarios humanos, se utiliza para evaluar la seguridad y eficacia de tratamientos o intervenciones contra enfermedades y problemas de salud de cualquier tipo, así como para determinar efectos de nuevos productos terapéuticos ${ }^{23}$.

Por lo anterior, la importancia de este estudio radica en el ECC, el cual es considerado como un paradigma de la investigación médica. Es decir, los ensayos clínicos son el camino óptimo para resolver un problema de investigación, por lo cual han revolucionado la práctica médica alrededor del mundo.

El conocer la evidencia científica existente permite mejorar la práctica clínica, así como identificar las necesidades y prioridades en la investigación.

En los últimos años se han realizado diversos estudios para identificar los ensayos clínicos publicados en diferentes áreas de las ciencias médicas ${ }^{24-27}$. Sin embargo, al momento, ninguno ha identificado los ensayos clínicos publicados en el BMHIM. Este reporte proporcionará información relevante para fomentar el uso de la evidencia científica existente, además de ofrecer un panorama general sobre los temas de estudio en los cuales aún existe un vacío de información.

El objetivo de este artículo fue identificar, describir y evaluar la calidad metodológica de los ECC publicados en el BMHIM.

\section{Métodos}

Se condujo un estudio observacional y descriptivo. Se diseñó una búsqueda sistemática de la literatura para identificar los ensayos clínicos publicados en el BMHIM. La búsqueda incluyó la exploración de la base de datos electrónica de la bibliohemeroteca del HIMFG, mediante el uso de la herramienta de búsqueda. También se realizaron búsquedas en el sitio web de la revista y se revisó cada uno de los números y volúmenes impresos del boletín ${ }^{28}$. Adicionalmente, se realizaron búsquedas en la base de datos de Elsevier ${ }^{9}$.

Se consideraron como artículos elegibles los estudios que cumplían los siguientes criterios:

- Estudios experimentales que comparaban tratamientos en seres humanos: prospectivos (las intervenciones fueron planificadas antes de realizarse el estudio); comparativos (se comparaban entre sí dos o más tratamientos o intervenciones); con o sin aleatorización (asignación de los participantes a cada intervención mediante un método aleatorio, donde la unidad de aleatorización podía ser individuos, grupos o partes del cuerpo).

- Las intervenciones podían ser de cualquier tipo: farmacológicas, quirúrgicas, diagnósticas, pronósticas, educativas, organizativas u otras.

Se excluyeron los estudios cuasiexperimentales, publicación de protocolos, aquellas secciones o reportes en los cuales únicamente se hacía referencia a un ensayo clínico, como editoriales y cartas al editor. Cuando se encontraron varios informes que hacían referencia al mismo estudio, se consideró solamente el informe original.

El procedimiento de búsqueda se realizó manualmente por dos revisores independientes. Cada revisor realizó la búsqueda en todos los artículos contenidos en cada uno de los números y volúmenes de la revista. La revisión se condujo por periodos de cinco años que abarcaban desde el número más actual al más antiguo. Cuando no se identificaron ensayos clínicos en un periodo de más de cinco años consecutivos, se detuvo la búsqueda. 
Las etapas que se siguieron para la identificación de un artículo como ensayo clínico fueron las siguientes: - Lectura del índice

- Localización de las palabras clave en el título

- Lectura del resumen

- Lectura del apartado de métodos y resultados.

Para la rápida localización de los estudios, se tuvieron en cuenta algunas palabras clave, tales como ensayo clínico (clinical trial), estudio controlado (controlled trial), ensayo cruzado (cross-over trial), estudio prospectivo, asignación aleatoria (aleatorización/randomized, random), cegamiento o enmascaramiento (blinded, blinding), ensayo clínico abierto, grupo control, placebo.

El registro del proceso de búsqueda se realizó mediante un formulario elaborado en Word, el cual concentraba los años y el total de estudios revisados, así como el número de estudios identificados en cada uno de los números de la revista. También se realizó un formulario individual para el registro de cada uno de los estudios identificados.

Una vez identificados, ambos revisores evaluaron de manera independiente la elegibilidad de cada uno de los estudios para su inclusión. Posteriormente, se extrajo la información necesaria sobre las características individuales de los estudios incluidos. Los datos se introdujeron en tablas previamente diseñadas en Microsoft Exce ${ }^{\circledR}($ Versión 2010). Se recabaron datos como año de publicación, volumen, autor principal, nombre del artículo, diseño, tipo de intervención, brazos de comparación, método de asignación al tratamiento, aleatorización, unidades de aleatorización (individuos o grupos), cegamiento, entre otros. Las patologías evaluadas en los estudios se tomaron en cuenta de acuerdo con la clasificación del CIE-10.

\section{Evaluación de la calidad de los estudios}

Para evaluar la calidad metodológica de los ensayos clínicos identificados, se utilizó la herramienta de riesgo de sesgo, diseñada por la Colaboración Cochra$n \mathrm{e}^{29}$. La herramienta permite evaluar de manera crítica un ensayo clínico. Es decir, indica el grado de rigor con el cual se realizó una investigación de acuerdo con los estándares más altos posibles. La fiabilidad de los resultados de un ensayo clínico aleatorizado depende del grado con el cual se hayan evitado las fuentes potenciales de sesgo durante su realización. Esta herramienta permite conocer la validez interna de un estudio o qué tan confiables son sus resultados.
La herramienta de riesgo de sesgo incluye una descripción y una valoración de siete dominios que abordan aspectos específicos del estudio (Tabla 1). Para efectos de la presente investigación, únicamente se consideraron cinco dominios para la evaluación (generación de la secuencia, ocultamiento de la asignación, cegamiento de los participantes y del personal, cegamiento de los evaluadores del resultado, datos de resultado incompletos), los cuales permitirán conocer cómo se realizó el estudio, lo que permitirá valorar su calidad metodológica.

La valoración para cada dominio incluyó la respuesta a una pregunta: Sí indicó un bajo riesgo de sesgo; No indicó un alto riesgo de sesgo; y Poco claro indicó falta de información o incertidumbre acerca del posible sesgo.

La evaluación se realizó a partir de los informes de los estudios individuales mediante dos revisores independientes. Cuando existieron desacuerdos entre los revisores, se resolvieron mediante una discusión y consenso o por un tercer revisor.

Una vez realizada la evaluación de los estudios, se desarrollaron gráficos que ilustran la proporción de estudios con cada una de sus evaluaciones ("bajo riesgo", "alto riesgo", "riesgo poco claro") y todas las evaluaciones en una tabulación cruzada por entrada.

\section{Análisis de datos}

Los resultados se presentaron de manera narrativa y gráfica e incluyó la descripción de los estudios identificados. La información se expresó mediante porcentajes, proporciones temporales y la proporción de la evaluación del riesgo de sesgo para cada dominio dentro de sus tres categorías (alto, bajo y no claro).

\section{Resultados}

Se revisaron mediante búsqueda manual 73 números, con 363 volúmenes del BMHIM. La revisión abarcó desde el año 1968 hasta diciembre de 2016 (volumen 25 a 73); es decir, un periodo de 48 años. Dentro del periodo de estudio se identificaron 4,925 artículos originales publicados, de los cuales únicamente el $1 \%$ correspondió a ensayos clínicos (67/4,925). El primer ensayo clínico publicado en el BMIHM se identificó en 1973 (volumen 30).

La mayor proporción de ensayos clínicos publicados se identificó durante el periodo de 1993 a 1997 (20/67 ECC; 30\%), seguido por el periodo de 1998 a 2002 (15/67 ECC; 22\%) (Figura 1). 
Tabla 1. Criterios contemplados para la evaluación de riesgo de sesgo

Dominio

Sesgo de selección

Generación de la secuencia

Ocultamiento de la asignación

Sesgo de realización

Cegamiento de los participantes y del personal. Se debería evaluar cada resultado principal (o cada clase de resultado).

Cegamiento de los evaluadores del resultado. Se debería evaluar cada resultado principal (o cada clase de resultado).

Sesgo de desgaste

Datos de resultado incompletos. Se debería evaluar cada resultado principal (o cada clase de resultado).

Sesgo de notificación

Notificación selectiva de los resultados. Se debería evaluar cada resultado principal (o cada clase de resultado).

Otros sesgos

Otras fuentes de sesgo

\section{Descripción}

Describe el método utilizado para generar la secuencia de asignación con detalle suficiente para permitir una evaluación de si la misma produjo grupos comparables.

Describe el método utilizado para ocultar la secuencia de asignación con detalle suficiente para determinar si las asignaciones a la intervención se podían prever antes o durante el reclutamiento.

Describe todas las medidas utilizadas, si se utilizó alguna, para cegar a los evaluadores del estudio al conocimiento de qué intervención recibió un participante. Proporcionar cualquier información con respecto a si el cegamiento propuesto fue efectivo. Describe todas las medidas utilizadas, si se utilizó alguna, para cegar a los participantes y al personal del estudio al conocimiento de qué intervención recibió un participante. Proporcionar cualquier información con respecto a si el cegamiento propuesto fue efectivo.

Describe la compleción de los datos de resultado para cada resultado principal, incluidos los abandonos y las exclusiones del análisis. Señalar si se describieron las/los abandonos y las exclusiones, los números en cada grupo de intervención (comparados con el total de participantes asignados al azar), los motivos de las deserciones/exclusiones cuando se detallaron, y cualquier reinclusión en los análisis realizados por los supervisores.

Señala como los revisores examinaron la posibilidad de la notificación selectiva de los resultados, y qué encontraron.

Señala alguna inquietud importante acerca del sesgo no abordado en los otros dominios del instrumento. Si en el protocolo de la revisión se preespecificaron preguntas/ítems particulares, se deberían proporcionar las respuestas para cada pregunta/ítem.

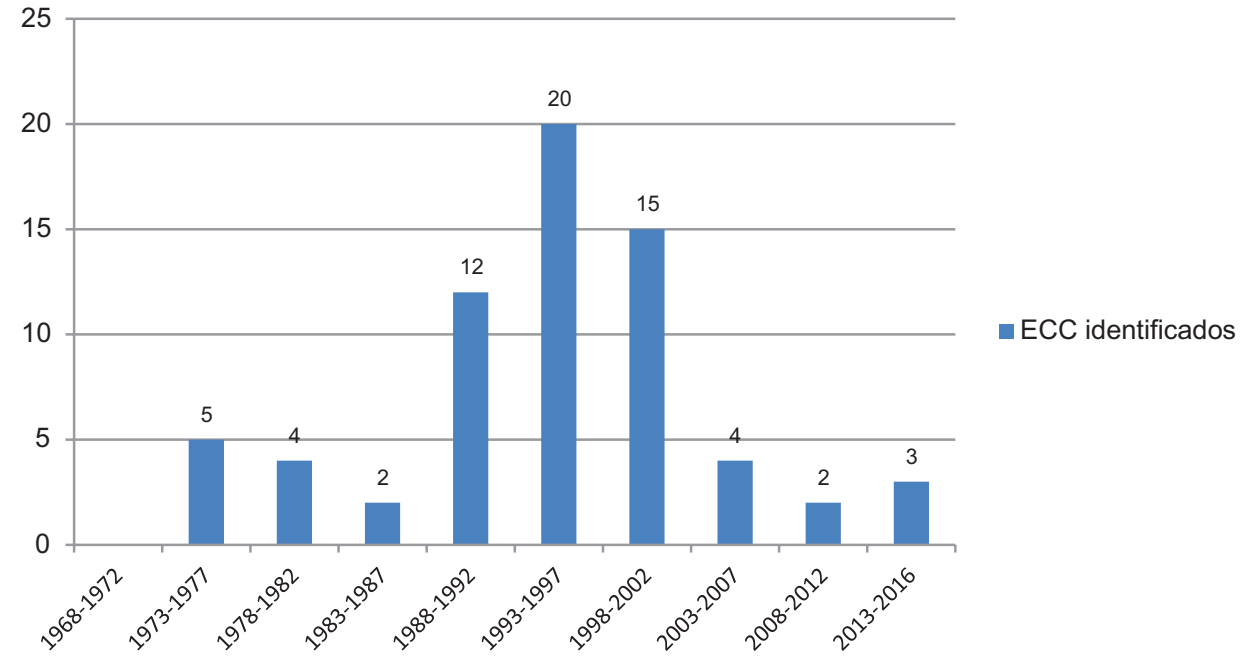

Figura 1. Ensayos clínicos publicados en el BMHIM en un periodo de 48 años.

ECC: ensayo clínico controlado. 


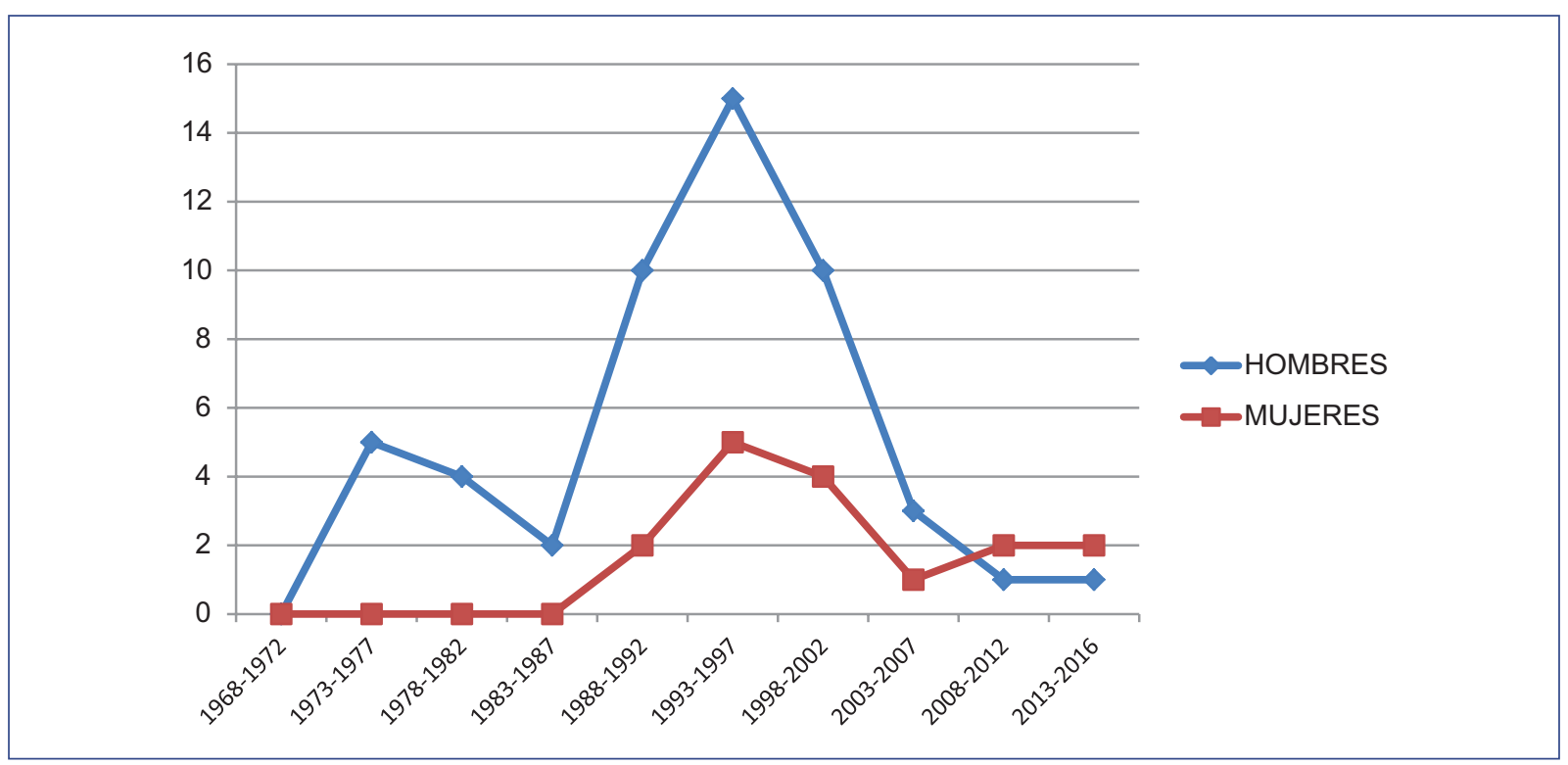

Figura 2. Relación hombre/mujer (autor principal) de los ensayos clínicos publicados en el BMHIM.

En las publicaciones identificadas se pudo observar que el número de autores fue variable, con predominio del rango que va desde tres a más de seis autores. Del mismo modo, prevaleció el sexo masculino de los autores catalogados como autor principal (52/67 ECC; $78 \%$ ) (Figura 2).

La mayoría de los ensayos clínicos se desarrollaron en el contexto nacional y en el tercer nivel de atención sanitaria (63/67 ECC; 94\%). Únicamente cinco estudios fueron desarrollados en otros países (Argentina, Chile, Venezuela y Cuba), y la mayor parte se llevaron a cabo en un solo centro (65/67 ECC; 97\%). Los estudios contemplan las diferentes especialidades y áreas médicas, con predominio de los estudios desarrollados en el área de hidratación oral (13/67 ECC; 19\%), neonatología (10/67 ECC; 15\%), infectología (7/67 ECC; 10\%), nutrición y neumología (6/67 ECC; 9\% en ambos casos).

Los ensayos clínicos relacionados con enfermedades infecciosas y parasitarias fueron los más frecuentemente publicados (17/67 ECC; $25 \%$ ), así como los ensayos sobre enfermedades endocrinas, nutricionales y metabólicas (8/67 ECC; 12\%). Todos los ensayos se realizaron en población pediátrica (0-18 años), predominando los estudios en lactantes (24/67 ECC; $35 \%$ ) y escolares (21/67 ECC; $31 \%)$. Únicamente en tres estudios no se describió el rango de edad de los participantes. El tamaño de muestra de los ensayos osciló entre 8 y 674 participantes. Las intervenciones evaluadas en los estudios fueron, en su mayoría, de tipo farmacológico
(58/67 ECC; 86\%). Solamente un estudio evaluó una intervención de tipo quirúrgico. Del total de estudios identificados, 10 ensayos clínicos utilizaron el placebo como control (Tabla 2).

Dentro de las características metodológicas de los ensayos clínicos, la gran mayoría contemplaba dos brazos de comparación (60/67 ECC; 89\%). Solamente un estudio evaluó más de tres brazos. Un gran número de los ensayos reportó haber realizado aleatorización (58/67 ECC; 86\%). Sin embargo, un escaso número reportó datos de enmascaramiento, ya fuera como simple (6/67 ECC; $9 \%$ ) o doble ciego (11/67 ECC; 16\%). Diecisiete ensayos clínicos (25\%) realizaron el análisis por intención de tratar. La mayoría de los estudios no reportaron la fuente de financiación (57/67 ECC; 85\%), ocho ensayos informaron haber recibido apoyo institucional y dos recibieron apoyo de la industria farmacéutica (Tabla 2).

Los resultados obtenidos sobre la calidad metodológica de los ensayos clínicos reportaron que, para la generación de la secuencia, 43 ECC (64\%) presentaron un riesgo de sesgo poco claro; para el dominio de ocultamiento de la asignación, 48 ensayos (72\%) presentaron alto riesgo de sesgo. En cuanto al cegamiento de los participantes y el personal, la mayoría de los ensayos clínicos (50/67 ECC; 75\%) obtuvieron un alto riesgo de sesgo, al igual que para el cegamiento de los evaluadores (51/67 ECC; 76\%). Finalmente, para el dominio de datos de resultados incompletos, el $45 \%$ de los estudios (30/67 ECC) presentó un bajo 
Bol Med Hosp Infant Mex. 2018;75

Tabla 2. Características de los ensayos clínicos identificados

Ensayos clínicos ( $n=67$ )

Variable

n (\%)

Lugar de elaboración

Nacional

$62(92)$

Internacional

5 (7)

Número de autores

Uno

Dos

$5(7)$

Tres

$19(28)$

Cuatro

$11(16)$

Cinco

$15(22)$

Seis o más autores

17 (25)

Autor principal en los estudios

Mujeres

$15(22)$

Hombres

Nivel de atención

Primer nivel

Segundo nivel

Tercer nivel

Número de centros participantes

Uno

65 (97)

Dos

Más de dos

Especialidad y área de estudio

Anestesiología

Cardiología

$1(1)$

Cirugía

Dermatología

1 (1)

Gastroenterología

2 (3)

Hidratación oral

$1(1)$

Infectología

13 (19)

7 (10)

Inmunología y Alergología

1 (1)

Nefrología

$5(7)$

Neonatología

10 (15)

Neumología

$6(9)$

Nutrición

6 (9)

Medicina familiar

2 (3)

Odontología

1 (1)

Oncología-Hematología

3 (4)

Unidad de cuidados intensivos neonatales

$4(6)$

Unidad de terapia intensiva pediátrica

$1(1)$

Patología (CIE-10)

Enfermedades congénitas del sistema nervioso central 1 (1)

Enfermedades de la piel y del tejido subcutáneo

Enfermedades de la sangre y de los órganos hematopoyéticos 3 (4)

Enfermedades del periodo perinatal

$7(10)$

$1(1)$

Enfermedades del sistema circulatorio

Enfermedades del sistema digestivo

Enfermedades del sistema genitourinario

$5(7)$

Enfermedades del sistema respiratorio

$7(10)$

8 (12)

Enfermedades endocrinas, nutricionales y metabólicas

17 (25)

Tumores (neoplasias)

No especificada

Tipo de población

Neonato o recién nacido (0-28 días)

Lactante (1 mes-2 años)

Preescolar (2-5 años)

Escolar (6-11 años)

Adolescente (12-18 años)

2 (3)

No especificada 
Tabla 2. Características de los ensayos clínicos identificados (continuación)

\section{Ensayos clínicos ( $n=67$ )}

Variable

n (\%)

Tamaño de muestra

8-647 participantes

Tipo de intervención

Farmacológica

No farmacológica

$8(86)$

Quirúrgica

$8(12)$

$1(1)$

Placebo

9 (14)

Brazos de comparación

2 brazos

60 (89)

3 brazos

6 (9)

Más de 3 brazos

$1(1)$

Aleatorización

Aleatorizado

$58(86)$

No aleatorizado

9 (13)

Unidades de aleatorización

Pacientes

66 (99)

Grupos de pacientes

Cegamiento

Simple

6 (9)

Doble

$11(16)$

Triple

$0(0)$

No especificado

$50(75)$

Análisis por intención de tratar

Fuente de financiación

Institucional

Industria farmacéutica

2 (3)

No especificada

$57(85)$

Registro en Clinical trials

1 (1)

riesgo de sesgo. En las figuras 3 y 4 se muestra el resumen de la evaluación de riesgo de sesgo de los estudios incluidos.

\section{Discusión}

En este estudio se presenta una perspectiva general de la producción de ensayos clínicos publicados en el BMHIM con el propósito de mostrar el crecimiento en el número de publicaciones y planificar estrategias para mejorar en el futuro. Es importante recalcar que el HIMFG cuenta con diversas áreas de investigación. Sin embargo, como se pudo observar, los ensayos clínicos publicados son escasos (en promedio 1.4 ensayos publicados/año). Incluso, es importante señalar que la publicación de ensayos clínicos en el BMHIM ha disminuido en la última década. Asimismo, a pesar de que el HIMFG es una institución con una alta concentración de población oncológica, los estudios que se han publicado en esa área aún son limitados.
El presente estudio también demostró que las mujeres representan escasamente el 22\% (15/67 ECC) del porcentaje de primeros autores de artículos de investigación original, mientras que los hombres tuvieron más del $70 \%$ de probabilidades de ser el primer autor de un ECC. En México se ha observado la desigualdad de género. De acuerdo con el último informe sobre brecha mundial de género, que analiza la división de los recursos y las oportunidades entre hombres y mujeres, México tiene una brecha de género del $70 \%$, lo cual refleja una gran desigualdad de género en la economía y el mundo laboral calificado, así como en la política, el acceso a la educación y la esperanza de vida. Aunque las disparidades de género han disminuido gradualmente durante las últimas décadas, se requieren más esfuerzos para lograr la igualdad de género. Sería deseable que estas diferencias alcanzaran, al menos, la media mundial $(32 \%)^{30}$.

Por otro lado, se observó que la mayoría de los ensayos clínicos publicados en el BMHIM presenta un alto riesgo de sesgo, principalmente en el ocultamiento 


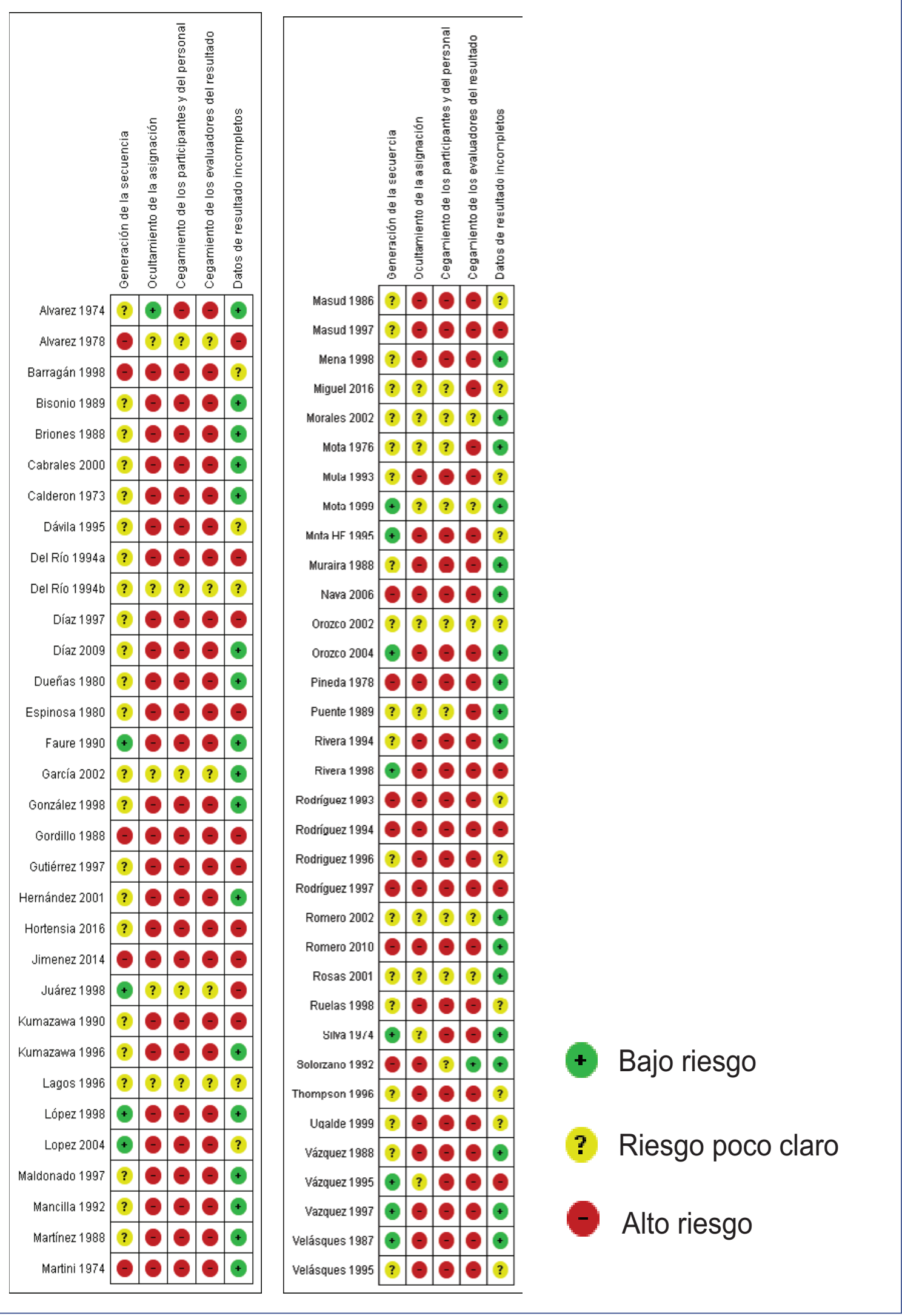

Figura 3. Resumen del riesgo de sesgo de los ensayos clínicos: Juicio de los autores sobre cada dominio de riesgo de sesgo para cada estudio identificado. 


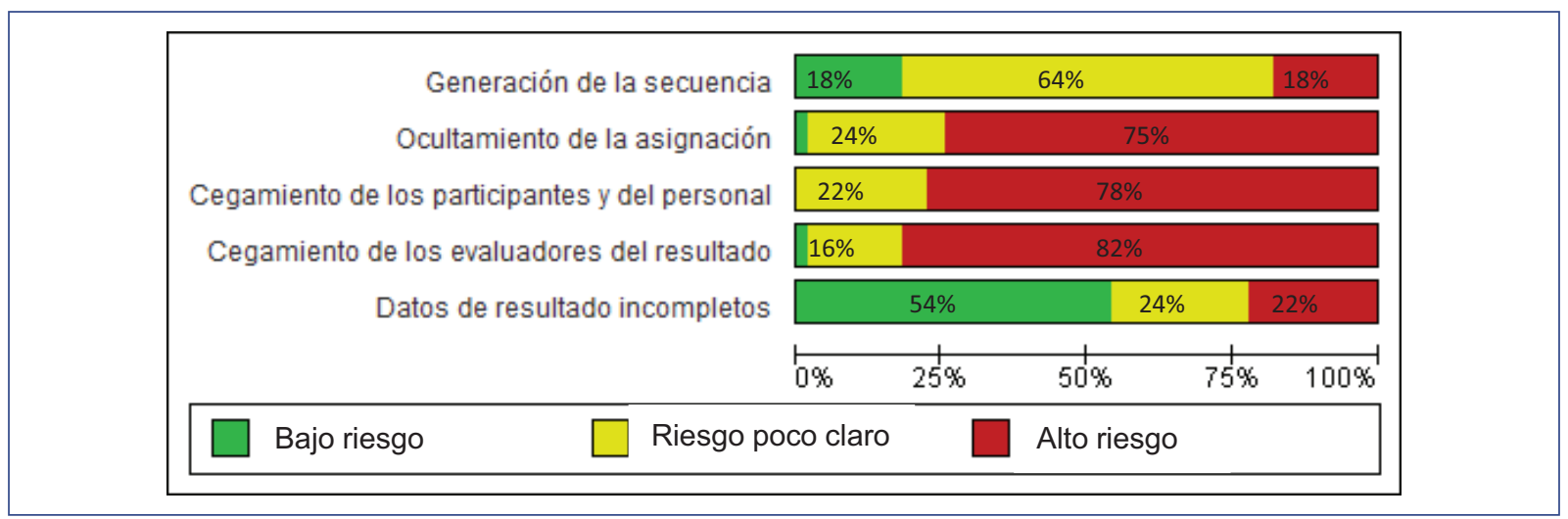

Figura 4. Gráfico de riesgo de sesgo global de los ensayos clínicos identificados.

de la asignación y el cegamiento, lo cual puede influir en la validez y confiabilidad de los resultados. Una de las fortalezas del ensayo clínico es la asignación al azar, la cual, de realizarse correctamente, evita el sesgo de selección en la asignación de las intervenciones a los participantes. Es importante asegurar el azar y evitar conocer de qué manera se produce la secuencia de la asignación: es decir, ocultar adecuadamente las asignaciones siguientes de los participantes del estudio; con ello se evitan fuentes potenciales de sesgo. Por otro lado, el adecuado cegamiento o enmascaramiento puede reducir el riesgo de que se afecten los resultados, además de garantizar que los grupos comparados reciban la misma atención y manejo ${ }^{29}$. El hecho de que un ensayo clínico posea una adecuada calidad metodológica asegura su validez interna y disminuye los sesgos, lo cual garantiza la confiabilidad de sus resultados y su aplicabilidad a la práctica clínica.

Diversos organismos internacionales han propuesto estrategias para mejorar la calidad y la transparencia en los estudios de investigación en salud. Dentro de estas iniciativas se encuentra la declaración CONSORT (Consolidated Standards of Reporting Trials), la cual pretende mejorar el reporte de los ensayos clínicos, facilitando a los autores la preparación de los informes $^{31-33}$. CONSORT se ha establecido como un estándar a seguir; sin embargo, en el estudio se pudo observar que la calidad de los ensayos clínicos no mejoró con el tiempo desde el establecimiento de CONSORT en 1999 y hasta la actualidad.

Se estima que aproximadamente el $50 \%$ de los ensayos clínicos realizados en el mundo no llegan a publicarse. La razón principal es el reporte de resultados negativos ${ }^{34}$. También se sabe que un gran número de ensayos clínicos nunca llega a registrarse en las bases de datos existentes ${ }^{35-37}$, lo cual se confirma en nuestro estudio, ya que únicamente se identificó un ensayo registrado en Clinical Trials. La falta de registro y publicación de ensayos clínicos impide conocer qué estudios se han desarrollado y cuáles fueron sus resultados. La iniciativa de All Trials promueve que todos los resultados de los ensayos clínicos sean reportados, sin importar si estos conllevan riesgos o beneficios ${ }^{38}$.

En la última década, la colaboración Cochrane se ha dedicado a identificar los ensayos clínicos existentes en el mundo para integrarlos en la base de datos de ensayos clínicos de The Cochrane Library (CENTRAL), todo ello con el fin de ofrecer la mayor cantidad de información disponible ${ }^{39,40}$. Es importante considerar que no todas las revistas que publican información científica se encuentran indexadas en las bases de datos bibliográficas ${ }^{41}$, 10 cual dificulta la identificación de los ensayos clínicos, y más aún la aplicabilidad de sus resultados. Sin embargo, el disponer de un registro de ensayos clínicos publicados en revistas indexadas y no indexadas garantiza su uso como evidencia disponible para la elaboración de guías de práctica clínica y revisiones sistemáticas, que a su vez son una herramienta útil para la toma de decisiones en salud $^{42}$. Este trabajo forma parte del proyecto de identificación de ensayos clínicos en revistas Iberoamericanas liderado por el Centro Cochrane Iberoamericano ${ }^{40,42}$.

Para concluir, se tiene que los ensayos clínicos publicados en el BMHIM aún son escasos y de baja calidad metodológica. Los futuros ensayos clínicos deberían apegarse a los criterios CONSORT para mejorar su calidad, y con ello disminuir los problemas derivados de información inadecuada.

Aún existen áreas de investigación clínica pediátrica en las cuales se requiere más iniciativa por parte de clínicos e investigadores para realizar más ensayos 
clínicos controlados, con la finalidad de crear mejores estrategias para la terapéutica de los pacientes, así como elevar la calidad de la investigación.

\section{Responsabilidades éticas}

Protección de personas y animales. Los autores declaran que para esta investigación no se han realizado experimentos en seres humanos ni en animales.

Confidencialidad de los datos. Los autores declaran que han seguido los protocolos de su centro de trabajo sobre la publicación de datos de pacientes.

Derecho a la privacidad y consentimiento informado. Los autores declaran que en este artículo no aparecen datos de pacientes.

\section{Conflicto de intereses}

Los autores declaran no tener ningún conflicto de intereses.

\section{Financiamiento}

Los autores no recibieron ningún tipo de financiamiento para la elaboración y desarrollo de este proyecto.

\section{Agradecimientos}

Agradecemos a Michelle Rojas Ramírez por su contribución en el proceso de revisión manual del BMHIM.

\section{Bibliografía}

1. Himfg.com.mx [Internet]. México: Hospital Infantil de México Federico Gómez. Disponible en: http://himfg.com.mx/interior/el_instituto.html.

2. Torregrosa L. Cincuenta años de pediatría en el Hospital Infantil de México. Gac Med Mex. 1993;129:351.

3. Kumate J. Federico Gómez Santos (1897-1980). Bol Med Hosp Infant Mex. 1996:53:301-2.

4. Chico PLF. Inauguración del Hospital Infantil de México. Bol Med Hosp Infant Mex. 2010;67:33-7.

5. Lizardo J. 70 aniversario del Hospital Infantil de México Federico Gómez. Rev Med Hondur. 2014;82:37-8.

6. López M, Ruíz M, Miranda S, Gamboa F, Varela C, Sarmiento F, et al. Los Institutos Nacionales de Salud. Secretaria de Salud. 2002;1:16-8.

7. Rodríguez S. Centenario del natalicio del Dr. Federico Gómez Santos. Rev Biomed. 1997;8:274-5.

8. García J, Medeiros M. Hospital Infantil de México. Semblanza. Rev Invest Clin. 2009;61:1-3.

9. Boletín Médico del Hospital Infantil de México [Internet]. México: Journal Elsevier. Disponible en: http://www.journals.elsevier.com/boletin-medico-del-hospital-infantil-de-mexico.

10. latindex.org [Internet]. México: Latindex. Disponible en: http://latindex. org/latindex/ficha?folio=360.

11. Conacyt.gob.mx [Internet]. México: Sistema de Clasificación de Revistas Mexicanas de Ciencia y Tecnología. Disponible en: http://www.revistascytconacyt.mx/resultados/225?area $=3$.

12. Ardanuy J. Breve introducción de la bibliometría. Barcelona: Universitat de Barcelona; 2012. Disponible en: http://diposit.ub.edu/dspace/bitstream/2445/30962/1/breve\%20introduccion\%20bibliometria.pdf.

13. Flores D. Resumen del sistema de clasificación Dewey. México: Universidad Nacional Autónoma de México; 2009.
14. Sackett DL, Straus SE, Richardson WS, Rosenberg W, Haynes RB. Evidence-based medicine. How to Practice and Teach EBM. Edinburg: Churchill Livingston; 2000. pp. 261.

15. Jadad AR, Enkin MW. Randomized controlled trials. Massachusetts: Blackwell Publishing. BMJ Books; 2007.

16. Moher D, Jadad AR, Nichol G, Penman M, Tugwell P, Walsh S. Assessing the quality of randomized controlled trials: an annotated bibliography of scales and checklists. Control Clin Trials. 1995;16:62-73.

17. Moreno M. La Medicina basada en la evidencia y la práctica médica. Rev Cubana de Med. 2005;44:3-4.

18. Sachett D, Rosemberg W. The need for evidence-based medicine. JR Soc Med. 1995;88:620-4.

19. Demirdjian G. Historia de los ensayos clínicos aleatorizados. Arch Argent Pediatr. 2006;104:5867.

20. Richmond C. Sir Richard Doll. BMJ. 2005;331:295

21. Díaz NJ, Gallego MB, León GA. Medicina basada en evidencias. Rev Cubana Med Gen Integr. 2000;16:366-9.

22. Tuells J, Arístegui J. Vacuna Salk de polio: el ensayo de campo de Thomas Francis Jr. Y el incidente Cutter. Universidad de Alicante. 2006;7:136-9.

23. Viramontes J, Lazcano E, Angeles A, Salazar E, Hernández A, Gutiérrez P. Ensayos clínicos aleatorizados: variantes, métodos de aleatorización, análisis, consideraciones éticas y regulación. Salud Publica Mex. 2004;46:559-84.

24. Marti J, Bonfill X, Urrutia G, Lacalle JR, Bravo R. The identification and description of clinical trials published in Spanish journals of general and internal medicine during the period of 1971-1995. Med Clin. 1999;112: 28-34.

25. Montori VM, Wang YG, Alonso-Coello P, Bhagra S. Systematic evaluation of the quality of randomized controlled trials in diabetes. Diabetes Care. 2006;29:1833-8.

26. Barajas-Nava LA, Calvache JA, López-Alcalde J, Solà I, Cosp XB. Identification and description of randomized controlled trials and systematic reviews on patient safety published in medical journals. J Patient Saf. 2013;9:79-86.

27. Calvache JA, Barajas-Nava L, Sánchez C, Giraldo A, Domingo AJ, Delgado-Noguera M. Risk of bias assessment of clinical trials published in the Revista Colombiana de Anestesiología. Rev Colomb Anestesiol. 2012;40:183-91.

28. Boletín Médico del Hospital Infantil de México [Internet]. Compendio Digital 1944-2014. Disponible en: http://187.237.57.42/bmhim/consulta. html.

29. Higgins JPT, Altman Sterne JAC. Chapter 8: Assessing Risk of Bias in Included Studies. En: Higgins JPT, Green S, editores. Cochrane handbook for systematic reviews of interventions version 5.1.0. The Cochrane Collaboration; 2011. Disponible en: www.cochrane-handbook.org.

30. Weforum.org [Internet]. World Economic Forum. Global Gender Gap Report 2017. Disponible en: https://www.weforum.org/reports/the-globalgender-gap-report-2017.

31. Zwarenstein M, Treweek S, Gagnier JJ, Altman DG, Tunis S, Haynes B, et al. Improving the reporting of pragmatic trials: an extension of the CONSORT statement. BMJ. 2008;337:a2390.

32. Moher D, Hopewell S, Schulz KF, Montori V, Gøtzsche PC, Devereaux PJ, et al. CONSORT 2010 Explanation and Elaboration: updated guidelines for reporting parallel group randomised trial. BMJ. 2010; 340:c869.

33. Equator-network.org. The EQUATOR Network [Internet]. 2006. Disponible en: http://www.equator-network.org.

34. Alfaro V. Ensayos clínicos, buena práctica de publicación y regulaciones legislativas. MedClin. 2004;123:100-3.

35. Trialscentral.org [Internet]. Your source for online clinical trials information. Disponible en: http://www.trialscentral.org.

36. clinicaltrials.gov [Internet]. Clinical Trials. Linking patients to medical research. Disponible en: http://www.clinicaltrials.gov.

37. Controlled-trials.com [Internet]. Current Controlled Trials. Featuring the Meta Register of controlled trials. Disponible en: http://www.controlled-trials.com.

38. Altrials.net [Internet]. 2016. Disponible en: http://www.alltrials.net.

39. Cochrane.org [Internet]. Cochrane Community. Cochrane Central Register of Controlled Trials (CENTRAL). Disponible en: http://community.cochrane.org/editorial-and-publishing-policy-resource/overview-cochrane-library-and-related-content/databases-included-cochrane-library/ cochrane-central-register-controlled-trials-central.

40. Cochrane.org [Internet]. Cochrane Iberoamerica. Proyecto de identificación de ensayos clínicos iberoamericanos. Disponible en: http://es. cochrane.org/es/proyecto-de-identificaci\%C3\%B3n-de-ensayos-cl\%C3\%ADnicos-iberoamericanos.

41. Bonfill X, Osorio D, Posso M, Solà I, Rada G, Torres A, et al. Identification of biomedical journals in Spain and Latin America. Health Info Libr J. 2015;32:276-86.

42. Pardo-Hernandez $H$, Urrútia $G$, Barajas-Nava LA, Buitrago-Garcia D, Garzón JV, Martínez-Zapata MJ, et al. BADERI: an online database to coordinate handsearching activities of controlled clinical trials for their potential inclusion in systematic reviews. Trials. 2017;18:273. 